\title{
PLANT SPECIES CLASSIFCATION USING BOF
}

\author{
Silpa Susan Alex \\ Computer Science and Engineering \\ Rajiv Gandhi Institute of Technology \\ Kottayam, Kerala, Indian
}

\begin{abstract}
Automated plant species identification system could help botanists and layman for identifying plant species expeditiously. In this research, a $\mathrm{BoF}(\mathrm{Bag}$ of Features) approach similar to that of deep learning is used to extract the features of the leaves and classify them hastily. 16 species are there in the dataset and in each species there are 30 images, so a total of $16 \times 30$, 480 images are in total. The leaf images are given as input and they undergoes different filtering techniques and segmentation, after that the image is fed into Bag of Features for feature extraction and classification. In olden days the shape, colour and the leaf vein morphometrics are used for feature extraction. Here the texture features of the leaves are used for extraction and classification. In addition to this, in this research the GLCM feature extraction method and Naive Bayes classification method is also used for comparing the result of Bag of Features approach. Using GLCM and Nave Bayes, get an accuracy of $89 \%$ in which in BoF 96\% accuracy is obtained. From the research, we can conclude that the BoF can be an effective automated system for plant species identification.
\end{abstract}

Keywords-BoF, GLCM, Naïve Bayes, Thresholding, Filtering, Segmentation.

\section{INTRODUCTION}

It is impossible or practically not possible for a layman or an expert, to be able to identify and classify all the plant species. In addition, some plant species may show high similarity between each other, taking a long time to categorize them. In addition, many plants face the problem of annihilation. Endangered and non-endangered plant species need to be maintained and conserved in a proper way to reduce the risks of extinction. Hence, there is a need to develop an automated or computerized system to identify and classify plants smoothly. Leaf shape is the most commonly used feature used to develop such automated plant classification systems. Other than shape, the leaf can provide additional information such as textures, veins, and colours.

With the progression of science and technology, machine learning has been widely employed for classification and recognition tasks in many domains especially in the biological fields. Machine learning techniques, such as, the Artificial Neural Network, Support Vector Machines, k-Nearest Neighbour, and others are artificial intelligent techniques mainly employed to perform pattern recognition and deep learning is impressive for feature extraction because it is better in providing deeper information of images. Deep learning is a machine learning technique that teaches computers to do what comes naturally to humans and in deep learning, a computer model learns to perform classification tasks directly from images, text, or sound.

In this project, $\mathrm{BoF}$ is applied to extract the features from leaf images of selected tree species and classify them. In another way also we are classifying the species, by using GLCM for feature extraction and the extracted features were then fed into a Naive Bayes approach for learning and training purposes. The gray-level co-occurrence matrix (GLCM), also known as the gray-level spatial dependence matrix which is used for feature extraction. In machine learning, naive Bayes classifiers are based on applying Bayes' theorem with strong (naive) independence assumptions between the features. The method in which we are using GLCM and Naive Bayes getting an accuracy of $89 \%$ and in BoF an improved accuracy of $96 \%$ is obtained.

Feature extraction involves simplifying the amount of resources required to describe a large set of data accurately. When performing investigation of complex information one of the serious issues originates from the quantity of factors included. Investigation with an enormous number of factors by and large requires a lot of memory and calculation control or an order calculation which over fits the preparation test and sums up ineffectively to new examples. Highlight extraction is a general term for techniques for building mixes of the factors to get around these issues while as yet depicting the information with adequate exactness. Surface material or visual normal for a surface. Surface examination points in finding a one of a kind method for speaking to the hidden qualities of surfaces and speak to them in some less complex however interesting structure, with the goal that they can be utilized for powerful, precise grouping and division of items. Though texture plays a significant role in image analysis and pattern recognition, only a few architectures implement onboard textural feature extraction.

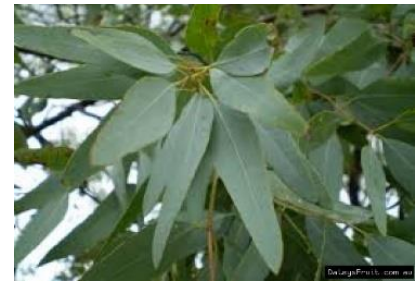

Figure 1: (a) Eucalyptus (b) Maavu

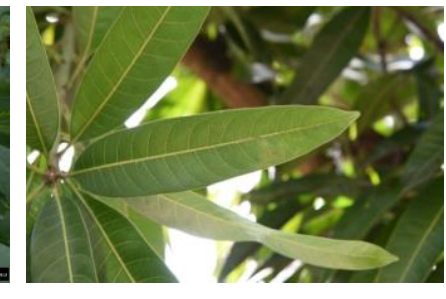




\section{International Journal of Engineering Applied Sciences and Technology, 2019 \\ Vol. 4, Issue 5, ISSN No. 2455-2143, Pages 124-132 \\ Published Online September 2019 in IJEAST (http://www.ijeast.com)}

The figure 1,2 are some of the leaves species that are utilized in my work. They have scientific names and here I referenced the normally known name in kerala. Here Eucalyptus (Eucalyptus Alba),Maavu(Mangifera Indica), Mandharam (Bauhinia Blakaena), Paala(Alstonia Scholaris), Manjadi(Adenanthera Pavonina) are metioned here. The 30 images of each species will be selected for feature extraction and classification purpose.

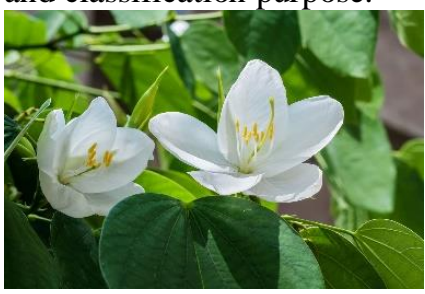

Figure 2: (a) Mandharam

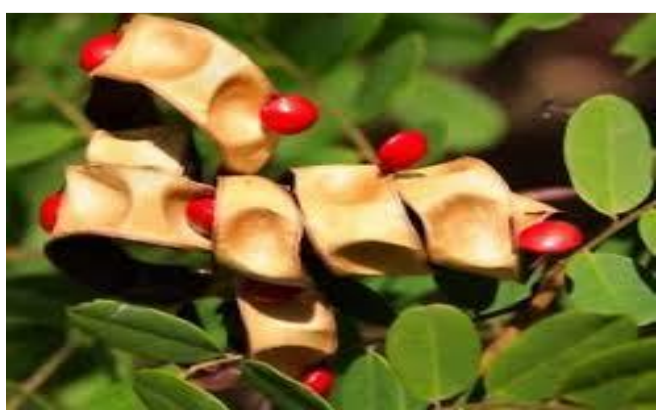

(c) Manjadi

For understanding the pictures effectively I incorporate the normal names. The accumulation of the pictures that we are utilized for the feature extraction technique is demonstrated as follows; figure: 3,4. The above demonstrated pictures are from outside and it can likewise be utilized for classification purposes. The texture features of the leaf images are used for feature extraction purposes.

Texture is significant component considered in an image processing and computer vision field that describes the surface and structure of a given object or region. Fundamentally, a picture is a blend of pixels and texture and is characterized as an entity having gathering of commonly related pixels inside a picture. Textures are an example of non-uniform spatial dispersion of varying picture powers, which core interest basically on the individual pixels that make up a picture. Surface is characterized by measuring the spatial connection between materials in a picture. Picture texture has various clear characteristics which play a significant role in depicting texture. Following properties are assuming an important role in unfurling texture: uniformity, regularity, density, linearity, directionality, direction, coarseness, roughness, phase and frequency.

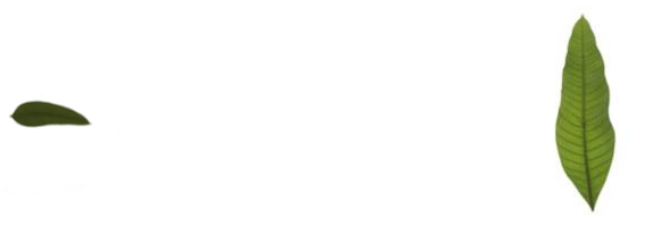

-

Figure 3: Mangifera indica, Eucalyptus alba, Adenanthera
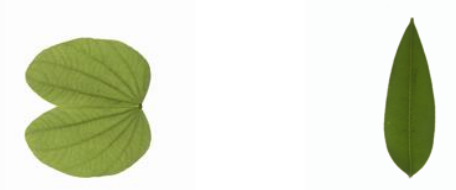

Figure 4: Bauhinia blakaena, Alstonia scholaris

The Gray Level Co-occurrence Matrix (GLCM) method is a way of extracting second order statistical texture features. The methodology has been utilized in various applications, Third and higher request surfaces think about the connections among at least three pixels. These are hypothetically conceivable yet not usually executed because of estimation time and understanding trouble. A GLCM is where the quantity of lines and segments is equivalent to the quantity of dim dimensions, $\mathrm{G}$, in the picture. The network component $\mathrm{P}(\mathrm{I}, \mathrm{j} \mid \Delta \mathrm{x}, \Delta \mathrm{y})$ is the relative recurrence with which two pixels, isolated by a pixel remove $(\Delta x, \Delta y)$, happen inside a given neighborhood, one with force 'I' and the other with power ' $\mathrm{j}$ '. The framework component $\mathrm{P}(\mathrm{I}, \mathrm{j}$ | $d, \theta)$ contains the second request likelihood esteems for changes between dark dimensions ' $\mathrm{I}$ ' and ' $\mathrm{j}$ ' at a specific removal separate $d$ and at a specific edge $(\Theta)$. Utilizing an enormous number of force levels $G$ suggests putting away a ton of brief information, for example a $\mathrm{G} \times \mathrm{G}$ framework for every blend of $(\Delta x, \Delta y)$ or $(d, \theta)$. Because of their enormous dimensionality, the GLCM's are touchy to the size of the surface examples on which they are evaluated. Subsequently, the quantity of dim dimensions is frequently diminished.

Naive Bayes, also known as Naive Bayes Classifiers are classifiers with the assumption that features are statistically independent of one another. Unlike many other classifiers which assume that, for a given class, there will be some correlation between features, naive Bayes explicitly models the features as conditionally independent given the class. While this may seem an overly simplistic (naive) restriction on the data, in practice naive Bayes is competitive with more sophisticated techniques and enjoys some theoretical support for its efficacy. Because of the independence assumption, naive Bayes classifiers are highly scalable and can quickly learn to use high dimensional features with 
limited training data. This is valuable for some genuine world datasets where the measure of information is little in correlation with the quantity of highlights for every individual bit of information, for example, discourse, content, and picture information. Examples of modern applications include spam filtering, automatic medical diagnoses, medical image processing, and vocal emotion recognition.

\section{BACKGROUND STUDY}

Fig 5 shows the basic steps for classifying a plant species. Initially, the leaf images are collected using digital camera, scanner or some other equipments. The images were then pre-processed to remove noise and improve the quality of the image. It is a necessary step to remove the image noises in order to highlight or enhance the important features of an image. After that we are performing different filtering techniques. Finally, the extracted features are fed to classification or recognition system. There by we can identify the species in which the leaf is belonging to.

Leaves are commonly used in plant species recognition due to their availability through-out the year. The useful features can be acquired from a single leaf are; shape, texture, Venation, pattern, and colour. Each of these features could be extracted by different methods. However, some approaches can be applied to obtain more than one feature, for example, Zernike Moment can be applied to obtain shape and texture feature Shape is the most common feature that have been used to develop plant identification systems.

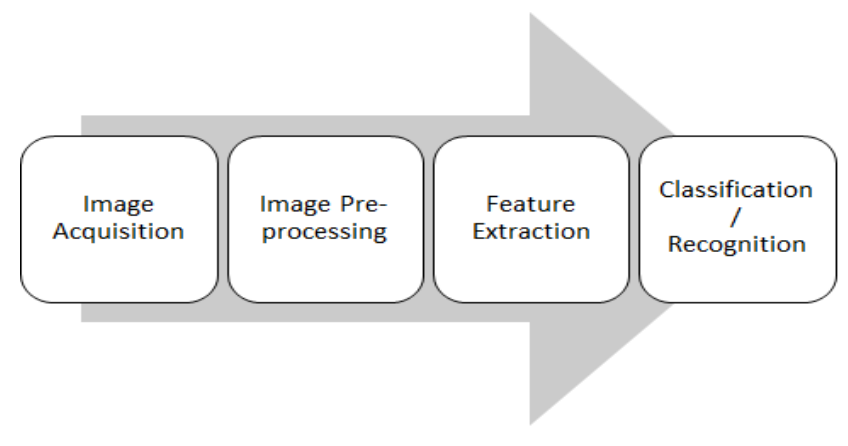

Figure 5: General Approach for Automated Plant Classification.

The features that are commonly used to evaluate shape are, ratio between slimness, roundness, compactness, rectangularity, and other aspects. Texture is one of the important features of the plant identification system, which can be used to characterize the leaves based on the surface structure of the leaves. It is a non-consistent spatial distribution pattern of different image intensities [8, 9], which concentrates mainly on each single pixel of an image. Venation pattern is another important feature for plant identification. Leaf veins can be further categorized into three types, which are primary veins, secondary veins and tertiary veins. Identification of plant species can also be done by using the colour of the leaves since leaf colour varies from species to species. Leaves from the same species may also be of different colours. For example, the young leaves of Cinnamomum inners is red in color which turn to green when they are matured.

\section{A. CONVENTIONAL APPROACH}

Cope et al. [10] introduced an evolved vein classifier based on genetic algorithms (GA) and Ant Colony algorithms to extract the vein structure. The evolved vein classifier is capable of extracting nearly the whole primary and secondary vein patterns with only a little noise. It was superior than the Ant Colony algorithms in extracting venation structures that are highly discontinuous. On the other hand, the Ant Colony algorithms, are more potent in continuous venation extraction, however, it may develop bigger and connected noise areas that may cause difficulty in actual vein identification. In short, the evolved classifiers out- performed the ant algorithms. Hence, a combination of both methods may be deemed to be more reliable.

Anami et al. [11] proposed A combination of colour and texture features based plant identfication system. The Sobel operator was used to extract the colour histogram and edge direction histogram as colour and texture features respectively from 1000 images of different types of herbs, shrubs and trees. The extracted features were then trained by using a radial basis exact _t neural network (RBENN) and a SVM.

Larese et al. [12] constructed an automated leaf identification approach for legumes based on the vein architecture only Simple measurements were applied on the vein morphology and then identified by a Random Forests approach. 39 vein features were extracted and then classiffied using the Random Forests. Furthermore, the authors discovered that the perfor- mance of using 7 features subset is comparable with the performance of 39 features.

Kadir et al. [13] proposed another method on the Foliage dataset and the Flavia dataset. The shape features, represented by 3 geometric features and the Polar Fourier Transform (PFT), colour moments, texture features extracted using GLCM and vein features were used to develop several models using different combination of features.

\section{B. BoF BASED PLANT IDENTIFICATION}

BoF the bag of features method is used for both feature extraction and classification. Ku-maraguru et al. [16] proposed Incidents or fire outbreaks are very common accidents occur-ring in Malaysia. The damage caused by this type of incident is very apocalypse towards nature and humans. Due to this fact, the need for fire detection application has been increased in recent years. In this paper we proposed a fire detection algorithm based on a combination of RGB and HSL filter to detect the color of the fire which is mainly comprehended by the intensity of the component $\mathrm{R}$ which is red color in this system. Then Bag-of-Features (BoF) classification model was used to classify and calculate the rate for fire present. The overall accuracy of this algorithm obtain is $98 \%$ and the efficiency is $89 \%$. The classification rate for the present of fire is 


\section{International Journal of Engineering Applied Sciences and Technology, 2019 \\ Vol. 4, Issue 5, ISSN No. 2455-2143, Pages 124-132 \\ Published Online September 2019 in IJEAST (http://www.ijeast.com)}

97.6\% Fire detection based on color filters and Bag-ofFeatures classification.

In Enhanced Bag-of-Features model for image classification by Lixia et al. [17] proposed that the feature representation and matching are two challenging problems for person e- identification problem. Designing a suitable feature representation method, and the ac- cording high efficiency matching scheme is meaningful. In this paper, a new person re- identification method was put forward. First, an improved BOF method was proposed, it use SURF algorithm to extract the preliminary feature and generate visual dictionary. And then, an effective classifier was designed by LIBSVM technology. This method can deal with illumination and scale invariants, and improve the efficiency of the matching process. The experimental results show that it is an effective scheme for person reidentification.

\section{METHODOLOGY}

This research consisted of four main steps as shown in Fig 5, which are, sampling, image pre-processing, feature extraction and classification. First, the leaf samples were collected and images were acquired. The leaf images were then pre-processed and fed into the feature extraction step to retrieve the important information from the leaves using CNN and Sobel edge detection approach. At last, the extracted features were trained and classified by using various machine learning methods.

\section{A. SAMPLING}

The leaf samples of this research were collected from three locations in the University of Malaya, Kuala Lumpur, Malaysia. These locations were areas around Varsity Lake (VL), main library (ML) and Dewan Tunku Canselor hall (DTC). The leaf images of this dataset were collected from the common tropical trees, which can be found easily in the University of Malaya as well as in Malaysia. The leaf is the part of the tree that is always chosen as the samples to be studied, instead of fruits, owers or some other parts due to its availability throughout the year. 16 species of tropical trees with 30 samples per species were collected. Thus, a total of 480 leaf images were collected for this research.

The leaf images were acquired by using a Nikon D750 model of DSLR camera. The samples were put on a box with white background and fluorescent lights were placed under the box in order to capture good quality images with standard background. As the lighting of this setup is from the bottom, it can help to reduce glaze and shadow on the leaf.

\section{B. IMAGE PRE-PROCESSING}

Raw images are not appropriate for analysis purposes and need to be converted into the processed format, such as, jpeg, jpg and tiff for further analysis. In this research, the accquired images were stored in a format of Nikon camera, named Nikon Electronic File (NEF) with 6016 x 4016 resolution. Adobe Photoshop was used to convert these raw images into
Tagged Image File Format (TIFF). In addition, the background noises of the images were reduced by using Adobe Photoshop and MATLAB R2016a was used for resizing and image conversion. Different pre-processing methods were applied namely; Filtering, Thresholding, Morphological Operation, Segmentation etc.

\section{Software and Hardware}

Workstation with Intel Xeon CPU E5-1603 v3 @ 2.80GHz processor, 64GB of RAM used execution of this research. MATLAB version 2016a and 2017b were used in feature extraction and classification.

\section{FEATURE EXTRACTION}

Feature extraction is the key stage in this research, which is used to extract the important features from the leaf images. The features that are commonly used for plant identification systems are shape [24, 25], texture [9, 24-26], colour [9, 24, $25]$ and vein [8, 24, 25, 27]. Various techniques can be utilized for feature extraction for example, Histogram of Situated Angle (Hoard), Zernike Minutes, Hu's Minute and others. A deep learning algorithm, namely, the Convolutional

Neural network, was employed to extract the features in this research. A conventional approach of using morphological feature extraction to segment the leaf venation by using Sobel edge detection method was explored and analyzed.

\section{a. Gray-Level Co-Occurrence Matrix (GLCM)}

A measurable technique for inspecting surface that considers the spatial relationship of pixels is the dark dimension co-event network (GLCM), otherwise called the dim dimension spatial reliance lattice. The GLCM capacities portray the surface of a picture by computing how frequently combines of pixel with explicit qualities and in a predetermined spatial relation-ship happen in a picture, making a GLCM, and after that removing factual measures from this network. Here we are considering 22 features Autocorrelation, Contrast, Correlation, Cluster Prominence, Cluster Shade, Dissimilarity, Energy, Entropy, Homogeneity, Maximum probability, Sum of sqaures, Sum average, Sum variance etc are some of the features that are selected for this work.

\section{b. Bag of Features (BoF)}

This technique is similar to that of Bag of words. In Bag of Word(BoW) textual information retrieval is considered and in Bag of feature(BoF) method image information retrieval is considered. For both feature extraction and classification; this BoF method can be used. Here in my work the texture of the leaf images are characterized by the repetition of basic elements or textons. From the image itself we can easily recognize that the features are bagged. Gathering the features of the leaves and that were utilized for clasifying or recognizing the leaf species effectively. 

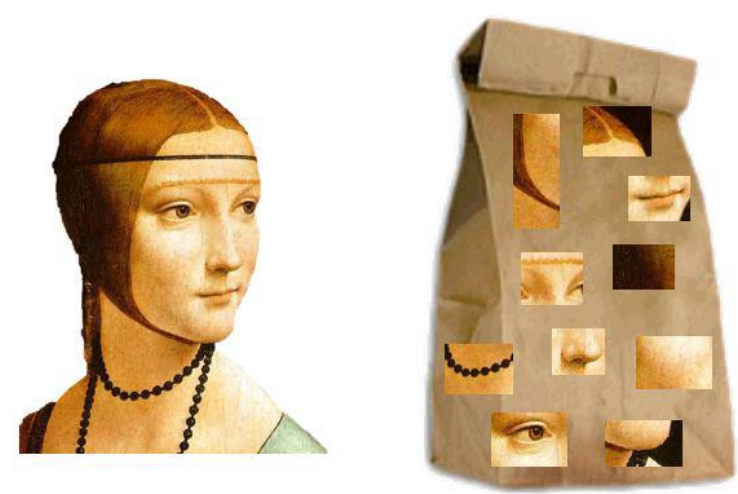

Figure 6: Bag of feature idea represcentation.

\section{CLASSIFIERS}

Classifiers incorporates an expansive scope of decisiontheoretic to deal with the distinguishing proof of pictures (or parts thereof). All characterization calculations depend on the suspicion that the picture being referred to portrays at least one highlights (e.g., geometric parts on account of an assembling arrangement framework, or ghastly areas on account of remote detecting, as appeared in the precedents underneath) and that every one of these highlights has a place with one of a few particular and selective classes. The classes might be indicated from the earlier by an examiner (as in supervised classification) or consequently bunched (for example as in unsupervised arrangement) into sets of model classes, where the investigator simply indicates the quantity of wanted classifications. (Classification and segmentation have firmly related destinations, as the previous is another type of part marking that can result in division of different highlights in a scene.)

\section{a. Naive Bayes}

Naive Bayes classifiers are an accumulation of classification algorithms based on Bayes Theorem. It is not a single algorithm but a family of algorithms where all of them share a common principle, that is every pair of features being classified is independent of each other. Here we are using Naive Bayes classifier and BoF for classification.

Bayesian classifier is a statistical classifier which attempts to make prediction of the class with an unknown sample based on probability [31]. NB assumes that all features of the samples are unrelated to each other [34]. However, the conditional independence of Bayes theorem diminishes the classification accuracy.

\section{b. Bag Of Features}

Classification, as the last step for an automated plant recognition system, is an intelligent algorithm in training data to recognize the specific features of each individual plant species and categorizing a new sample as the correct species. The favoured machine learning methods for plant identification are Artificial Neural Network (ANN), Support Vector Machines (SVM) and k-Nearest Neighbour (kNN). Usually the classification methods that was used in the research were SVM, ANN, kNN, Nave Bayes (NB) and CNN.
Support Vector Machines (SVM), a supervised machine learning approach, is conceded as one of the powerful classification methods due to its high capability in dealing with high dimensional space and data points which are not linearly separated [31]. Applying linear SVM on feature-mapped data can execute speedy with low storage and improve the classification performance [14]. Linear SVM with One versus all scheme was employed in this research since it involves a multi-classes dataset.

Artificial Neural Network (ANN) is the most common and frequent algorithm that have been applied in numerous researches $[3,13,15,17,32]$. The development of ANN was inspired by the humans brain neurons structure and behaviours. Different number of neurons and hidden layers in an ANN model may affect the classification performance. The proposed research employed a feed forward neural network with a single hidden layer which consisted of 80 neurons. The training process used the default Scaled Conjugate Gradient function and the achievement of a minimum gradient as the stopping criteria.

\section{IMPLEMENTATION}

\section{A. BLOCK DIAGRAM}

This exploration comprises of four fundamental strides as appeared in Fig 7, which are, sampling, image preprocessing, feature extraction and classification. In the first place, the leaf tests were gathered and pictures were procured. The leaf pictures were then pre- processed and fed into the feature extraction step to retrieve the important information from the leaves using BoF method. At last, the extracted features were trained and classified by using BoF. Making the result for comparison we are using the Gray Level Co-Occurance Matrix(GLCM) for another feature extraction and for classification the Naive Bayes is used.

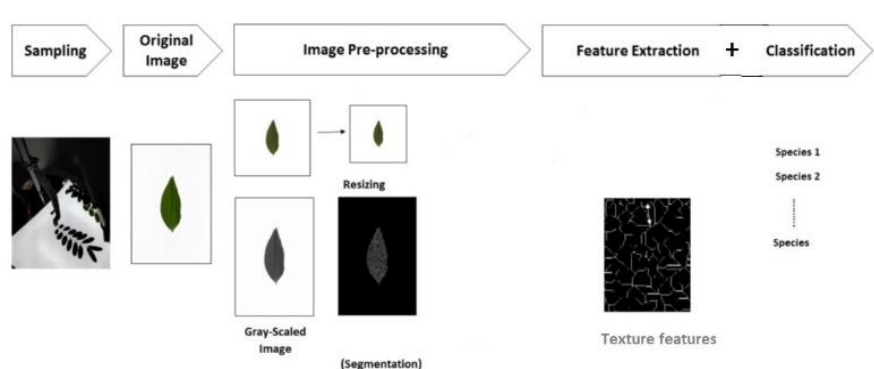

Figure 7: Proposed Block Diagram.

\section{B. SAMPLING}

This is the image acquistion stage. The leaf tests of this examination were gathered from three locations in the University of Malaya, Kuala Lumpur, Malaysia. These locations were areas around Varsity Lake (VL), main library (ML) and Dewan Tunku Canselor hall (DTC). The leaf pictures of this dataset were gathered from the regular tropical trees, which can be found effectively in the College of Malaya just as in Malaysia. The leaf is the piece of the tree that is dependably picked as the examples to be considered, rather than organic products, blooms or some different parts because of its accessibility consistently. 16 
types of tropical trees with 30 tests for every species were gathered. In this way, a sum of 480 leaf pictures were gathered for this research.

The leaf pictures were procured by utilizing a Nikon D750 model of DSLR camera. The examples were put on a container with white foundation and bright lights were set under the case so as to catch great quality pictures with standard foundation. As the lighting of this setup is from the bottom, it can help to reduce glaze and shadow on the leaf.

\section{IMAGE PREPROCESSING}

Raw images are not appropriate for analysis purposes and need to be converted into the processed format, such as, jpeg, jpg and tiff for further analysis. In this research, the accquired images were stored in a format of Nikon camera, named Nikon Electronic File (NEF) with 6016 x 4016 resolution. Adobe Photoshop was used to convert these raw images into Tagged Image File Format (TIFF). In addition, the background noises of the images were reduced by using MATLAB R2017 was used for resizing and image conversion. Different pre-processing methods were applied namely; Filtering, Thresholding, Morphological Operation, Segmentation.

\section{a. Filtering}

When an image is acquired by a camera or other imaging system, frequently the vision framework for which it is planned is unit to utilize it straightforwardly. The image may be corrupted by random variations in intensity, variations in illumination, or poor contrast that must be dealt with in the early stages of vision processing. Image filtering methods for image enhancement aimed at eliminating these undesirable characteristics. Image filtering is useful for many applications, including smoothing, sharpening, removing noise, and edge detection.

Most images are affected to some extent by noise, that is unexplained variation in data: disturbances in image intensity which are either uninterpretable or not of intrigue. Picture investigation is regularly improved if this noise can be sifted through. In an analogous way filters are used in chemistry to free liquids from suspended impurities by passing them through a layer of sand or charcoal. Engineers working in signal processing have extended the meaning of the term filter to include operations which accentuate features of interest in data. Employing this broader definition, image filters may be used to emphasize edges that is, boundaries between objects or parts of objects in images. Filters give a guide to visual understanding of pictures, and can likewise be utilized as an antecedent to encourage advanced preparing, for example, division.

\section{b. Segmentation}

Image segmentation is a usually utilized system in computerized picture handling and examination to parcel a picture into numerous parts or districts, frequently dependent on the qualities of the pixels in the picture. Picture division could include isolating closer view from foundation, or grouping districts of pixels dependent on similitudes fit as a fiddle.

\section{c. Thresholding}

After performing filtering into the input image we are performing thresholding. Image thresholding is a simple, yet effective, way of partitioning an image into a foreground and background. This image analysis technique is a sort of picture division that disengages questions by changing over grayscale pictures into paired pictures. Image thresholding is best in pictures with large amounts of differentiation. Common image thresholding algorithms include histogram and multilevel thresholding. Here we are performing linear thresholding to the filtered image.

\section{d. Morphological Operation}

Binary images may contain numerous imperfections. Specifically, the parallel districts created by straightforward thresholding are twisted by clamor and surface. Morphological picture handling seeks after the objectives of expelling these defects by representing the structure and structure of the picture. These procedures can be stretched out to greyscale images. The most fundamental morphological tasks are dilation and erosion. Dilation adds pixels to the limits of articles in a picture, while disintegration expels pixels on item limits. The quantity of pixels included or expelled from the articles in a picture relies upon the size and state of the organizing component used to process the picture.

\section{FEATURE EXTRACTION}

Feature extraction a kind of dimensionality decrease that proficiently speaks to intriguing pieces of a picture as a smaller element vector. This methodology is helpful when picture sizes are huge and a decreased element portrayal is required to rapidly finish assignments, Feature extraction, and matching are frequently consolidated common computer vision problems such as object detection and recognition, content-based image retrieval, face detection and recognition, and texture classification. Here for feature extraction we are using GLCM and BoF. Using GLCM features around 22 are collected. During BoF feature extraction the texture features of the images are extracted.

\section{E. RECOGNITION}

This is the classification stage and here for classification the Naive Bayes and BoF are used. The images that undergoes feature extraction are fed into classification, after performing training and testing we can easily classify the images of leaf in which species it belongs.

\section{EXPERIMENTAL RESULTS}

\section{A. GLCM AND NAIVE BAYES}

\section{Feature Extraction Using GLCM}

In the method of classifying plant species using GLCM for feature extraction and Naïve Bayes for classification; we have to train and test the available images in te dataset. We have a total of 480 images and we need to train that all images during classification. Before that we need to collect the features from the leaf images. Here we are using GLCM 


\section{International Journal of Engineering Applied Sciences and Technology, 2019 \\ Vol. 4, Issue 5, ISSN No. 2455-2143, Pages 124-132 \\ Published Online September 2019 in IJEAST (http://www.ijeast.com)}

the Gray Level Co-Occurrence Matrix for feature extraction. The method uses 22 features and the features are: Uniformity, Angular Second Moment, Entropy, Dissimilarity, Contrast, Inverse difference, correlation, Homogeneity, Autocorrelation, Cluster Shade, Cluster, Prominence, Maximum probability, Sum of Squares, Sum Average, Sum Variance, Sum Entropy, Difference variance, Difference entropy, Information measures of correlation, Information measures of correlation, Maximal correlation coefficient, Inverse difference normalized, Inverse difference moment normalized. These features are extracted from each leaf and on the basis of that the leaves are classsified after testing. The figure 8 shows the feature values obtained for different leaves. A to $\mathrm{V}$ shows the 22 features and the below are the values of each species. The column W shows the species classes. 1 for first class 2 for the second class and so on. In a single class there are 30 images belongs. From the image we can see the values of different features based on the leaf category.

\section{B. BAG OF FEATURES}

\section{a. Training}

In $\mathrm{BoF}$ the feature extraction and classification are performed together. Features of the input images after filtering and other morphological operations are extracted and using these features we are training the images. The figure: 12 shows that the label and count. Level means the different classses or different category species the image shows only 8 classes, and the count shows that the number of leaves in each label that is each species we are taking 30 images so the column count shows 30 for all the species.

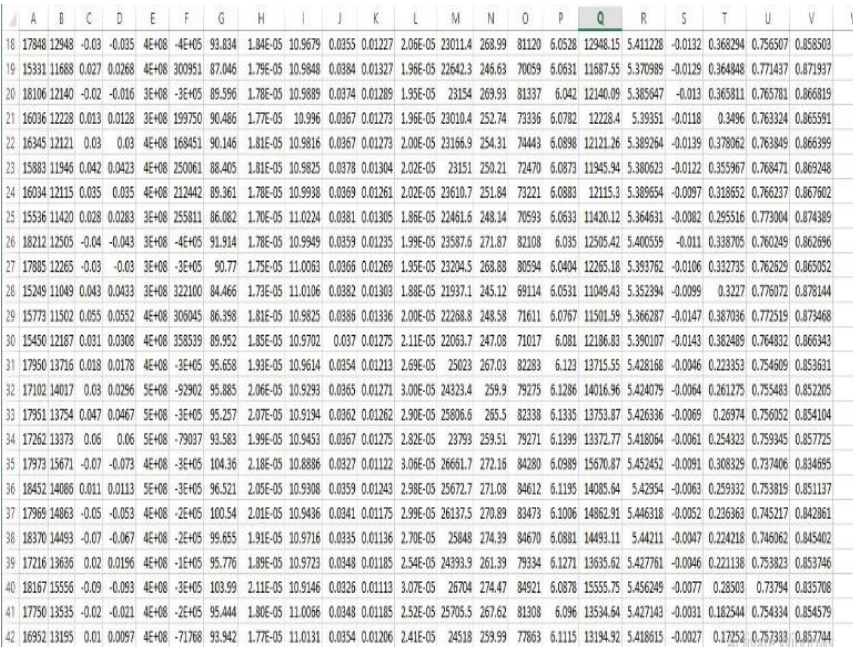

Figure 8: Extracted feature values from GLCM.

The training accuracy obtained from the $\mathrm{BoF}$ method is shown in figure: 13 the each row shows the known species and values and column shows the predicted ones. If we predicted the leaf as the correct one then it having the value 1.0. If it is corrected wrong having value 0.0 the values in between 1.0 to 0.0 shows the value range from correct to wrong. In the below an average accuracy is also calculated and shown that is 0.96 that is $96 \%$.
Label

\section{Count}

$\begin{array}{ll}\text { ACACIA } & 30 \\ \text { ADENANTHERA } & 30 \\ \text { ALSTONIA } & 30 \\ \text { BARRINGTONIA } & 30 \\ \text { BAUHINIA } & 30 \\ \text { EUCALYPTUS } & 30 \\ \text { FILICIUM } & 30 \\ \text { MANGIFERA } & 30\end{array}$

Figure 9: Trained images.

\section{b. Testing}

In beginning phase of testing result using Bag of Feature is shown below; the output of trained image is used as input for

\begin{tabular}{|c|c|c|c|c|c|c|c|c|}
\hline \multirow[b]{2}{*}{ KNOONS } & \multicolumn{8}{|c|}{ FREDICTED } \\
\hline & I ACACIA & ADENANTHERA & ALSTONIA & BARRINGTONIA & Bauhinia & EUCALYPTUS & FILICIOM & MANGIEEBA \\
\hline ACACIA & 1.00 & 0.00 & 0.00 & 0.00 & 0.00 & 0.00 & 0.00 & 0.00 \\
\hline ADENANTHERA & 10.00 & 1.00 & 0.00 & 0.00 & 0.00 & 0.00 & 0.00 & 0.00 \\
\hline ALSTONIA & 10.00 & 0.00 & 0.90 & 0.00 & 0.00 & 0.00 & 0.10 & 0.00 \\
\hline BARRINGTONIA & 0.05 & 0,00 & 0,00 & 0.81 & 0.00 & 0.00 & 0.00 & 0.14 \\
\hline BAUHINIA & 0.00 & 0.00 & 0,00 & 0.00 & 1.00 & 0.00 & 0.00 & 0.00 \\
\hline EUCALYYTUS & 10.00 & 0.00 & 0.00 & 0.00 & 0.00 & 1.00 & 0.00 & 0.00 \\
\hline FILTCTOM & 0.00 & 0.00 & 0.00 & 0.00 & 0.00 & 0.00 & 1.00 & 0.00 \\
\hline MANGIFERA & 0.00 & 0.00 & 0.00 & 0.00 & 0.00 & 0.00 & 0.05 & 0.95 \\
\hline
\end{tabular}

* Average Accuracy is 0.96 .

Figure 10: Training dataset.

testing the image in which species it belongs to. Figure: 11 shows the images in a single class that is the class ACACIA AURICULIFORMIS. As explained earlier, each species have 30 images, here the actual images is predicted as that same class so that the actual and predicted are showing same in the image. Here in the classification the 30 images in Acacia are correctly classified. 


\begin{tabular}{|c|c|c|c|}
\hline \multicolumn{4}{|c|}{ 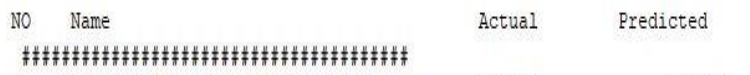 } \\
\hline 1 & seg-ACACIA AURICULIFORMIS 1.tif & ACACIA & ACACIA \\
\hline 2 & seg-ACACIA AURICULIFORMIS 10.tif & ACACIA & ACACIA \\
\hline 3 & seg-ACACIA AURICULIFORMIS 11.tif & ACACIA & ACACIA \\
\hline 4 & seg-ACACIA AURICULIFORMIS 12.tif & ACACIA & ACACIA \\
\hline 5 & seg-ACACIA AURICULIFORMIS 13.tif & ACACIA & ACACIA \\
\hline 6 & seg-ACACIA AURICULIFORMIS 14.tif & ACACIA & ACACIA \\
\hline 7 & seg-ACACIA AURICULIFORMIS 15 .tif & ACACIA & ACACIA \\
\hline 8 & seg-ACACIA AURICULIFORMIS 16. tif & $\mathrm{ACACIA}$ & ACACIA \\
\hline 9 & seg-ACACIA AURICULIFORMIS 17 .tif & ACACIA & ACACIA \\
\hline 10 & seg-ACACIA AURICULIFORMIS 18.tif & ACACIA & ACACIA \\
\hline 11 & seg-ACACIA AURICULIFORMIS 19.tif & ACACIA & ACACIA \\
\hline 12 & seg-ACACIA AURICULIFORMIS 2.tif & ACACIA & ACACIA \\
\hline 13 & seg-ACACIA AURICULIFORMIS 20.tif & ACACIA & ACACIA \\
\hline 14 & seg-ACACIA AURICULIFORMIS 21.tif & ACACIA & ACACIA \\
\hline 15 & seg-ACACIA AURICULIFORMIS 22.tif & ACACIA & ACACIA \\
\hline 16 & seg-ACACIA AURICULIFORMIS 23.tif & ACACIA & ACACIA \\
\hline 17 & seg-ACACIA AURICULIFORMIS 24.tif & ACACIA & ACACIA \\
\hline 18 & seg-ACACIA AURICULIFORMIS 25.tif & ACACIA & ACACIA \\
\hline 19 & seg-ACACIA AURICULIFORMIS 26.tif & ACACIA & ACACIA \\
\hline 20 & seg-ACACIA AURICULIFORMIS 27.tif & ACACIA & ACACIA \\
\hline 21 & seg-ACACIA AURICULIFORMIS 28.tif & ACACIA & ACACIA \\
\hline 22 & seg-ACACIA AURICULIFORMIS 29.tif & ACACIA & ACACIA \\
\hline 23 & seg-ACACIA AURICULIFORMIS 3.tif & ACACIA & ACACIA \\
\hline 24 & seg-ACACIA AURICULIFORMIS 30.tif & ACACIA & ACACIA \\
\hline 25 & seg-ACACIA AURICULIFORMIS 4, tif & ACACIA & ACACIA \\
\hline
\end{tabular}

Figure 11: Testing using BoF.

The figure:12 shows the confusion matrix. A confusion matrix is a table that is frequently used to portray the exhibition of a grouping model (or "classifier") on a lot of test information for which the genuine qualities are known. Here in the confusion matrix it represents the presence of 16 species. In row the actual value and column the predicted are shown. For example in the first class if we are looking the row 1 and column 1 together we get 30 that is all the images are correctly predicted that is our class Acacia. In the case of the class 3, actually we have 30 images but only the 28 are predicted correctly the remaining 2 belongs to the class 7; that is in 30 images 28 are correctly classified as class 3 and 2 images are wrongly belongs to the class species 7 .

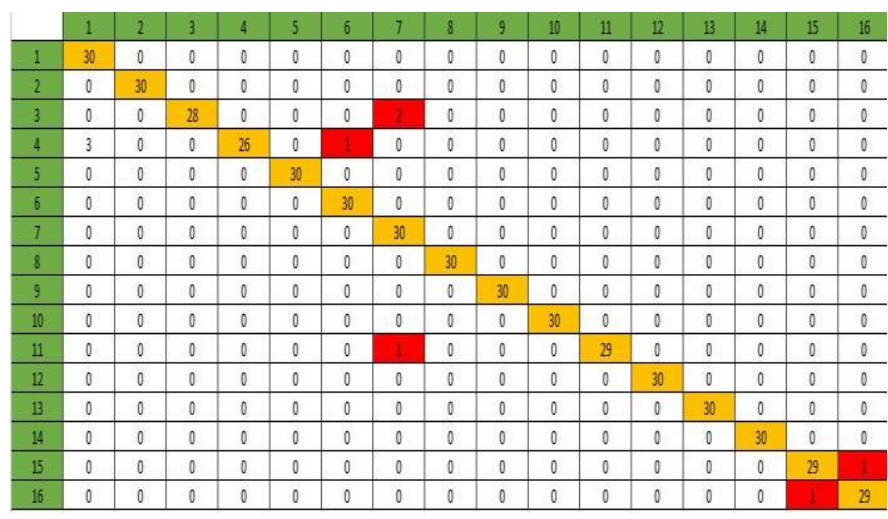

Figure 12: Confusion Matrix.

\section{c. Recognition}

In the stage of recognition the images are recognized in which species it belongs to. The figure: 13 shows the image of plant Acacia. As input we given the image of acacia and after training and testing, during the stage of recognition they classified as acacia itself, and the name is shown on the picture itself for easy identification or checking the classification easily.

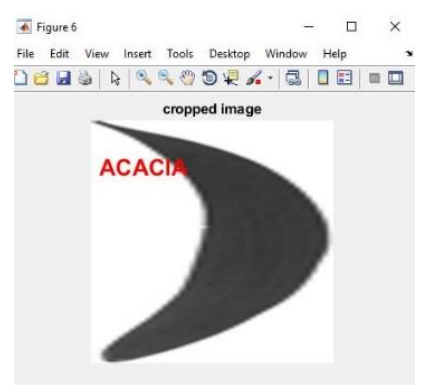

Figure 13: Species Recognition.

The table 1 below shows the comparison of the accuracy values of different algorithms. Same algorithms also shows different accuracy because of the different feature extraction method that is used. From the table the NB(Naïve Bayes) has accuracy $81.86 \%$ in [35] and 89 in this work. So from this table itself we can easily recognize that feature extraction method is also a factor influencing the accuracy of classification.

\begin{tabular}{|l|l|}
\hline Algorithm & Accuracy \\
\hline$[35]$ SVM & $82.75 \%$ \\
\hline$[35]$ NB & $81.86 \%$ \\
\hline NB & $89 \%$ \\
\hline BoF & $96 \%$ \\
\hline
\end{tabular}

Table 1: Accuracy Comparison.

\begin{tabular}{|l|l|l|l|}
\hline Algorithm & Accuracy & Sensitivity & Specificity \\
\hline BoF & $96 \%$ & $95.36 \%$ & $95.79 \%$ \\
\hline
\end{tabular}

Table 2: Accuracy, Sensitivity and Specificity of BoF.

\section{CONCLUSION}

In this work, we can conclude that the $\mathrm{BoF}$ is better than GLCM and Naive Bayes method for feature extraction and classification in plant species. More pre-processing works need to be done when using the conventional methods as compared to the BoF. Besides that, $\mathrm{BoF}$ is found to be a favourable feature extraction and classification method in this research. When using the $\mathrm{BoF}$ for feature extraction and classification, achieving a testing accuracy of $96 \%$. were Naive Bayes gives only $89 \%$. BoF method can be used for automated plant species classification.

\section{APPLICATIONS AND FUTURE SCOPE}

- It can be applied in the area of agriculture.

- Can be used in the area of unmanned shopping. 


\section{International Journal of Engineering Applied Sciences and Technology, 2019 \\ Vol. 4, Issue 5, ISSN No. 2455-2143, Pages 124-132 \\ Published Online September 2019 in IJEAST (http://www.ijeast.com)}

- Medicinal research.

- Botanical garden.

As a future work of this research, we would like to include more tropical plant species, especially those with compound leaves, and add in more taxonomic features to further enhance the plant species identification process that can be used by botanists and the public and we can also generate a phylogenic tree from this result.

\section{ACKNOWLEDGMENT}

This research was supported by the [35] Jing Wei Tan, Siow-Wee Chang, Sameem Abdul-Kareem et al., "Deep Learning for Plant Species Classification using Leaf Vein Morphometric ," IEEE/ACM Transactions on Computational Biology and Bioinformatics , 2018. and the date set is also taken from this paper.

\section{REFERENCES}

[1] Willis, K.J. (ed.) 2017. State of the World's Plants 2017. Report. Royal Botanic Gardens, Kew.

[2] Krizhevsky, A., Sutskever, I., \& Hinton, G. E. (2012). Imagenet classification with deep convolutional neural networks. In Advances in neural information processing systems (pp. 1097-1105).

[3] Wang, Z. (2015). The applications of deep learning on traffic identification. BlackHat USA.

[4] Wang, D., Khosla, A., Gargeya, R., Irshad, H., \& Beck, A. H. (2016). Deep learning for identifying metastatic breast cancer. arXiv preprint arXiv:1606.05718.

[5] Hinton, G., Deng, L., Yu, D., Dahl, G. E., Mohamed, A. R., Jaitly, N., Senior, A., Vanhoucke, V., Nguyen, P., Sainath, T.N. \& Kingsbury, B. (2012). Deep neural networks for acoustic modeling in speech recognition: The shared views of four research groups. IEEE Signal Processing Magazine, 29(6), 82-97.

[6] Goodfellow, I., Bengio, Y., \& Courville, A. (2016). Deep learning. MIT Press.

[7] Malladi, R., \& Sethian, J. A. (1996). A unified approach to noise removal, image enhancement, and shape recovery. IEEE Transactions on Image Processing, 5(11), 1554-1568

[8] , V., \& Ghorpade, J. (2013). An overview of the research on texture based plant leaf classification. arXiv preprint arXiv: 1306.4345.

[9] Arun, C., Emmanuel, W. S., \& Durairaj, D. C. (2013). Texture feature extraction for identification of medicinal plants and comparison of different classifiers. International Journal of Computer Applications, 62(12).

[10] Cope, J. S., Remagnino, P., Barman, S., \& Wilkin, P. (2010, December). The extraction of venation from leaf images by evolved vein classifiers and ant colony algorithms. In International Conference on Advanced Concepts for Intelligent Vision Systems (pp. 135-144). Springer Berlin Heidelberg.

[11] Anami, B. S., Suvarna, S. N., \& Govardhan, A. (2010). A combined color, texture and edge features based approach for identification and classification of indian medicinal plants. International Journal of Computer Applications,6(12), 45-51

[12] Larese, M., Craviotto, R., Arango, M., Gallo, C., \& Granitto, P. (2012). Legume identification by leaf vein images classification. Progress in Pattern Recognition, Image Analysis, Computer Vision, and Applications, 447-454.

[13] Kadir, A., Nugroho, L. E., Susanto, A., \& Santosa, P. I. (2013). Neural network application on foliage plant identification. arXiv preprint arXiv:1311.5829.

[14] Sulc, M., \& Matas, J. (2014). Texture-Based Leaf Identification. Paper presented at the European Conference on Computer Vision.

[15] Lee, S. H., Chang, Y. L., Chan, C. S., \& Remagnino, P. (2016). Plant identification system based on a convolutional neural network for the lifeclef 2016 plant classification task. In Working notes of CLEF 2016 conference.

[16] Lee, S. H., Chang, Y. L., Chan, C. S., \& Remagnino, P. (2016). Plant identification system based on a convolutional neural network for the lifeclef 2016 plant classification task. In Working notes of CLEF 2016 conference.

[17] Lee, S. H., Chan, C. S., Mayo, S. J., \& Remagnino, P. (2017). How deep learning extracts and learns leaf features for plant classification. Pattern Recognition, $71,1-13$

[18] Sladojevic, S., Arsenovic, M., Anderla, A., Culibrk, D., \& Stefanovic, D. (2016). Deep Neural Networks Based Recognition of Plant Diseases by Leaf Image Classification. Computational Intelligence and Neuroscience, 2016.

[19] Grinblat, G. L., Uzal, L. C., Larese, M. G., \& Granitto, P. M. (2016). Deep learning for plant identification using vein morphological patterns. Computers and Electronics in Agriculture, 127, 418-424.

[20] Larese, M. G., Namías, R., Craviotto, R. M., Arango, M. R., Gallo, C., \& Granitto, P. M. (2014). Automatic classification of legumes using leaf vein image features. Pattern Recognition, 47(1), 158-168.

[21] Reyes, A. K., Caicedo, J. C., \& Camargo, J. E. (2015). Fine-tuning Deep Convolutional Networks for Plant Recognition. In CLEF (Working Notes).

[22] Gupta, S., \& Mazumdar, S. G. (2013). Sobel edge detection algorithm. International journal of computer science and management Research, 2(2), 1578-1583.

[23] Vincent, O. R., \& Folorunso, O. (2009, June). A descriptive algorithm for sobel image edge detection. In Proceedings of Informing Science \& IT Education Conference (InSITE) (Vol. 40, pp. 97-107).

[24] Yadav, A. R., Dewal, M., Anand, R., \& Gupta, S. Classification of hardwood species.

[25] Prasvita, D. S., \& Herdiyeni, Y. (2013). MedLeaf: Mobile Application for Medicinal Plant Identification Based on Leaf Image. International Journal on 


\section{International Journal of Engineering Applied Sciences and Technology, 2019 \\ Vol. 4, Issue 5, ISSN No. 2455-2143, Pages 124-132 \\ Published Online September 2019 in IJEAST (http://www.ijeast.com)}

Advanced Science, Engineering and Information Technology, 3(2), 103-106.

[26] Ehsanirad, A., \& Sharath Kumar, Y. (2010). Leaf recognition for plant classification using GLCM and PCA methods. Oriental Journal of Computer Science and Technology, 3(1), 31-36.

[27] Aakif, A., \& Khan, M. F. (2015). Automatic classification of plants based on their leaves. Biosystems Engineering, 139, 66-75.

[28] Vedaldi, A. and Lenc, K. (2015) MatConvNet: Convolutional Neural Networks for MATLAB. Proceedings of the 23rd ACM International Conference on Multimedia, Brisbane, 26-30 October 2015, 689692. http://dx.doi.org/10.1145/2733373.2807412.26.

[29] DeepDreamImage. Retrieved March 10, 2017, from https://www.mathworks.com/help/nnet/ref/alexnet.html

[30] Convolution2dLayer. Retrieved March 10, 2017, from https://www.mathworks.com/help/nnet/ref/trainingoptio ns.html

[31] Caglayan, A., Guclu, O., \& Can, A. B. (2013, September). A plant recognition approach using shape and color features in leaf images. In International Conference on Image Analysis and Processing (pp. 161-170). Springer Berlin Heidelberg.

[32] Chang, S. W., Kareem, S. A., Kallarakkal, T. G., Merican, A. F. M. A., Abraham, M. T., \& Zain, R. B. (2011). Feature selection methods for optimizing clinicopathologic input variables in oral cancer prognosis. Asian Pac J Cancer Prev, 12, 2659-64.

[33] Pornpanomchai, C., Rimdusit, S., Tanasap, P., \& Chaiyod, C. (2011). Thai herb leaf image recognition system (THLIRS). Kasetsart J. (Nat. Sci.), 45, 551-562.

[34] McCallum, A., \& Nigam, K. (1998, July). A comparison of event models for naive bayes text classification. In AAAI-98 workshop on learning for text categorization (Vol. 752, pp. 41-48).

[35] Jing Wei Tan, Siow-Wee Chang, Sameem AbdulKareem et al., "Deep Learning for Plant Species Classification using Leaf Vein Morphometric ," IEEE/ACM Transactions on Computational Biology and Bioinformatics, 2018. 